\title{
Characteristics of Patients with Diabetes Initiating Sodium Glucose Co-transporter-2 Inhibitors (SGLT2i): Real-World Results from Three Administrative Databases in Japan
}

Yuichiro Ito · James Van Schyndle - Takuya Nishimura •

Toshifumi Sugitani · Tomomi Kimura

Received: December 20, 2018 / Published online: February 7, 2019

(C) The Author(s) 2019

\section{ABSTRACT}

Introduction: The aim of this study was to evaluate the characteristics of new users of sodium glucose co-transporter-2 inhibitors (SGLT2i) in comparison with those of new users of other oral antidiabetic drugs (OADs) using data retrieved from three administrative databases in Japan.

Methods: This study included adult patients from each database who started an OAD between 2014 and 2017. Outpatients who started SGLT2i therapy were included in the SGLT2i cohort. The remaining outpatients were grouped according to the OAD class of their earliest initial prescription after no use of the index OAD during the 6-month pre-index period. Diabetes-related complications were evaluated using the Diabetes Complication Severity Index.

Results: In total, 176,355 patients in the hospital-based administrative database (H-dataset),

Enhanced Digital Features To view enhanced digital features for this article go to https://doi.org/10.6084/ m9.figshare.7613837.

Electronic supplementary material The online version of this article (https://doi.org/10.1007/s13300019-0577-7) contains supplementary material, which is available to authorized users.

Y. Ito · T. Nishimura $\cdot$ T. Sugitani · T. Kimura ( $₫)$

Astellas Pharma Inc., Tokyo, Japan

e-mail: tomomi.kimura@astellas.com

J. Van Schyndle

Astellas US, LLC., Northbrook, IL, USA
98,361 in the pharmacy claims database (Pdataset) and 37,786 in the insurance claims database (I-dataset) were analyzed. In the H-dataset, SGLT2i users, compared with users of other OADs, tended to be younger (mean age at index: 57.7 vs. $60.3-69.2$ years) and to have a higher prevalence of hypercholesterolemia (73.5 vs. $55.2-71.4 \%$ ), a higher mean body weight (74.4 vs. $60.5-70.8 \mathrm{~kg})$, a higher body mass index (27.6 vs. $23.5-26.4 \mathrm{~kg} / \mathrm{m}^{2}$ ) and a higher glycated hemoglobin level (8.4 vs. $7.4-8.1 \%)$. There were no distinct differences in the prevalence of complications between SGLT2i users and users of other OADs in the H-dataset. Similar trends were noted in the other datasets.

Conclusion: Patients initiating SGLT2i therapy differed in several characteristics from new users of other OADs. SGLT2i were prescribed more frequently to younger patients, those at increased cardiovascular risk or those with poorer glycemic control.

Funding: Astellas Pharma Inc., Tokyo, Japan.

Keywords: Administrative databases; SGLT2i; Japan; Patient profile; Real-world

\section{INTRODUCTION}

The prevalence of diabetes among the adult population in Japan was estimated to be $7.7 \%$ in 2017 , and the expectation is that it will reach 
$9.8 \%$ in $2030[1,2]$. Patients with type 2 diabetes mellitus (T2DM) are often associated with clustered risk factors for cardiovascular disease (CVD), such as obesity, hypertension and hypercholesterolemia, and are also at increased risk of macrovascular complications [3]. In addition, microvascular complications, such as diabetic nephropathy, neuropathy and retinopathy, are also common in patients with T2DM [4].

Oral antidiabetic drugs (OADs) lower blood glucose levels through various mechanisms, such as by increasing insulin release [e.g. sulfonylureas (SU) and glinides], improving insulin sensitivity [e.g. thiazolidinediones (TZD)], stimulating glucose uptake via $5^{\prime}$ adenosine monophosphate-activated protein kinase activation [e.g. biguanides (BG)], delaying glucose absorption in the intestines [e.g. alpha-glucosidase inhibitors $(\alpha-\mathrm{GI})]$ and stimulating insulin release and suppressing glucagon secretion via dipeptidyl peptidase-4 (DPP-4) inhibition [e.g. DPP-4 inhibitors (DPP-4i)] [5, 6].

Sodium glucose co-transporter-2 inhibitors (SGLT2i) represent a novel class of OADs that reduce blood glucose levels by inhibiting renal glucose reabsorption, independent of insulin secretion and action [7]. Six SGLT2i compounds, namely ipragliflozin, dapagliflozin, tofogliflozin, luseogliflozin, canagliflozin and empagliflozin, were approved for the treatment of T2DM in Japan between April 2014 and February 2015 [8-14]. However, real-world studies on SGLT2i users in Japan are limited [15-18]. The aim of this study was to provide a real-world perspective on the demographic and clinical characteristics of SGLT2i users compared with those of users of other OADs using data retrieved from three administrative databases in Japan.

\section{METHODS}

This was a retrospective database analysis of patients with diabetes who initiated OAD therapy between April 2014 and March 2017 in Japan. The Medical Affairs Japan Protocol Review Committee reviewed and approved the study protocol prior to study initiation. As the study only involved analysis of pre-existing data in the databases, written informed consent from the study participants was not required. This study was conducted in accordance with the guidelines for Good Pharmacoepidemiology Practice [19].

\section{Data Sources}

This study retrieved patient data from three distinct administrative databases in Japan: (1) a hospital-based administrative database (H-dataset) constructed from data on inpatients and outpatients from 287 Diagnosis Procedure Combination (DPC) hospitals [20]; (2) a pharmacy claims database (P-dataset) comprising data from over 800 pharmacies nation-wide, which provided coverage for approximately $2 \%$ of all outpatient prescriptions [21]; and (3) an insurance claims database (I-dataset) containing medical and prescription claims of 3.8 million employees and their dependents (as of study execution) who were mostly aged $\leq 65$ years [22].

\section{Study Population}

This study included patients from each database who were $\geq 18$ years old as of 1 April 2014 and initiated therapy on an OAD between 1 April 2014 and 31 March 2017 (study period) (Fig. 1). Patients who had any of the following International Classification of Diseases tenth revision (ICD-10) diagnosis codes during the study period were excluded: E10.xx (type 1 diabetes mellitus), E12.xx (malnutrition-related diabetes mellitus), E13.xx (other specified diabetes mellitus) and O24.xx (diabetes mellitus in pregnancy). Patients with any SGLT2i prescription were identified, and the first medication date (= index date) was noted. Patients who initiated treatment with SGLT2i and any other OAD(s) or with $\geq 2$ SGLT2i agents on the same date were excluded from the study. The remaining patients, consisting of those who added SGLT2i to their prior OAD therapy or those who started SGLT2i monotherapy, were included in the SGLT2i cohort.

Since the other OADs included in this study for comparison have been in the market for a long time, we tried to include new users during 


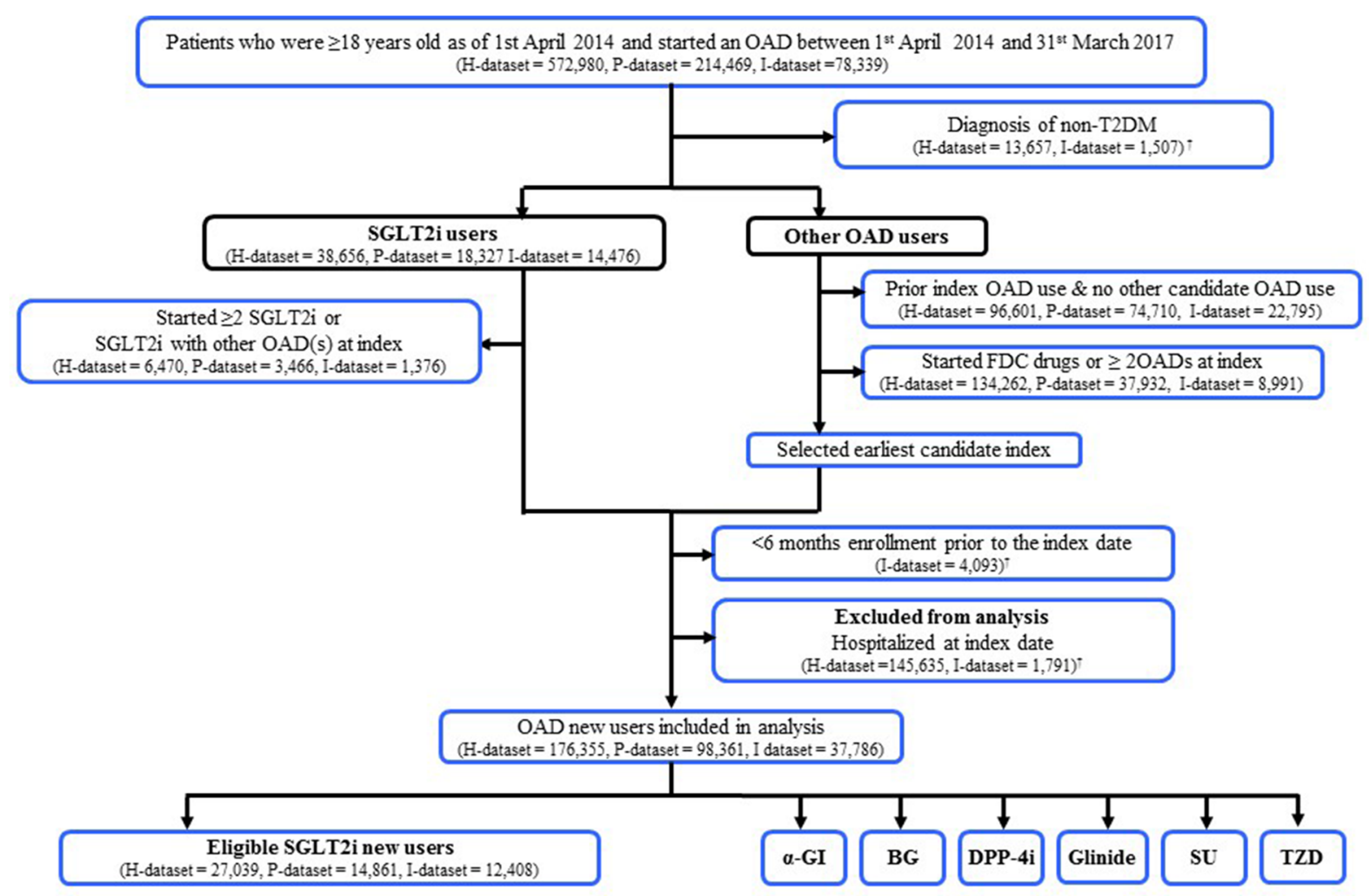

Fig. 1 Selection algorithm for the study population. ${ }^{\dagger}$ Due to the different nature of the datasets, certain exclusion criteria were only applicable to the stated dataset. $H$ dataset a hospital-based administrative database constructed from data for inpatients and outpatients from 287 diagnosis procedure combination (DPC) hospitals. $P$ dataset a pharmacy claims database using data from over 800 pharmacies nation-wide which provided a coverage of approximately $2 \%$ of all outpatient prescriptions. I-dataset

the study period to allow better comparison with the SGLT2i cohort. Other OAD cohorts in this study included patients receiving therapy with $\alpha$ GI, BG, DPP-4i, glinides, SU, and TZD [see Electronic Supplementary Material (ESM) Table 1 for a list of medication codes]. We identified the first prescription date for each OAD class and flagged this date as a candidate index date. If the patients used the index OAD during the 6-month pre-index period or initiated therapy with the index $\mathrm{OAD}$ together with another class of OADs or fixed-dose combination drugs on the same date (co-initiation), then the candidate index OAD was excluded. Finally, the earliest candidate was an insurance claims database containing medical and prescription claims of 3.8 million employees and their dependents that were mostly aged $\leq 65$ years. $\alpha$-GI alphaglucosidase inhibitors, $B G$ biguanides, $D P P-4 i$ dipeptidyl peptidase-4 inhibitors, $F D C$ fixed-dose combination, $O A D$ oral antidiabetic drug, $S G L T 2 i$ sodium glucose co-transporter-2 inhibitors, $S U$ sulfonylureas, T2DM type 2 diabetes mellitus, $T Z D$ thiazolidinediones

selected as the index $\mathrm{OAD}$, and the initial prescription date for the index OAD was identified as the index date. Patients who had $<6$ months enrollment prior to the index date were excluded from the study cohorts (only applicable to the I-dataset). For better generalizability, patients who were hospitalized at the index date were excluded from the analysis (only applicable to the $\mathrm{H}$ - and I-datasets).

\section{Study Assessments}

We evaluated patient characteristics and prescribing site characteristics for the SGLT2i and 


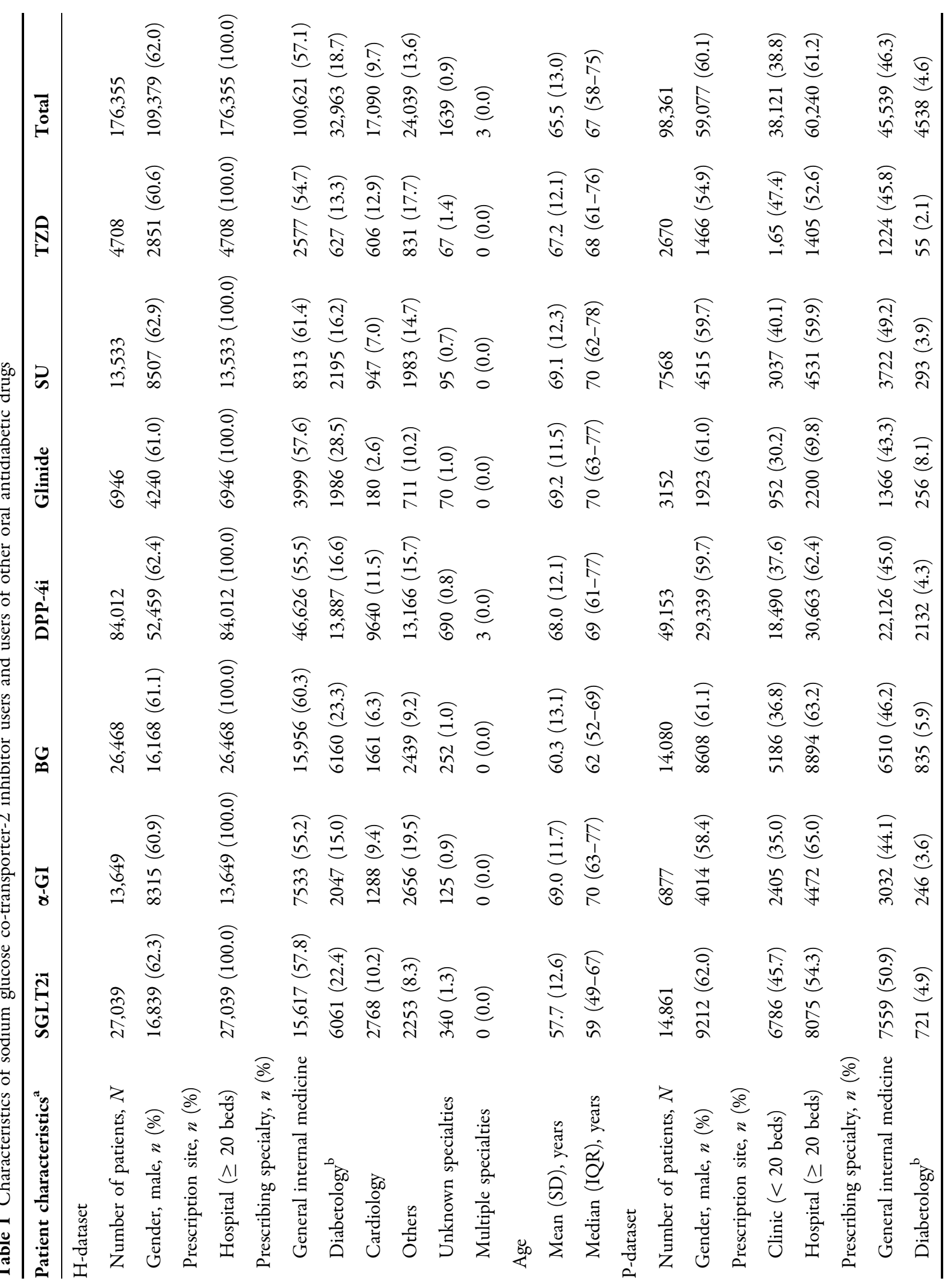




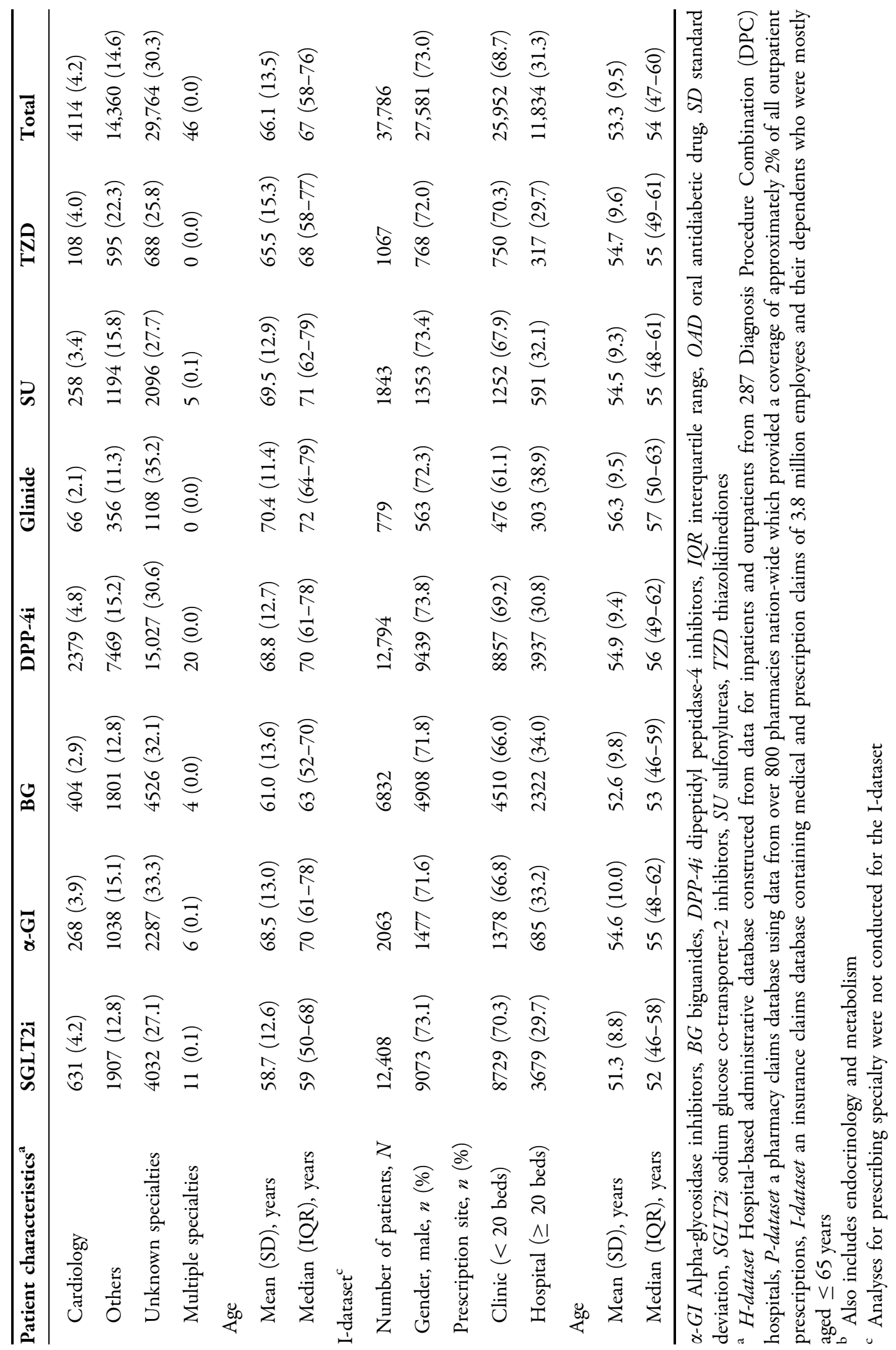




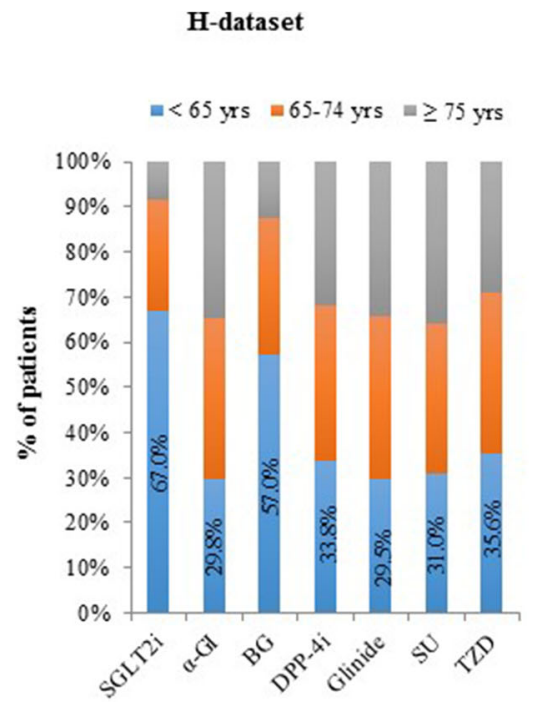

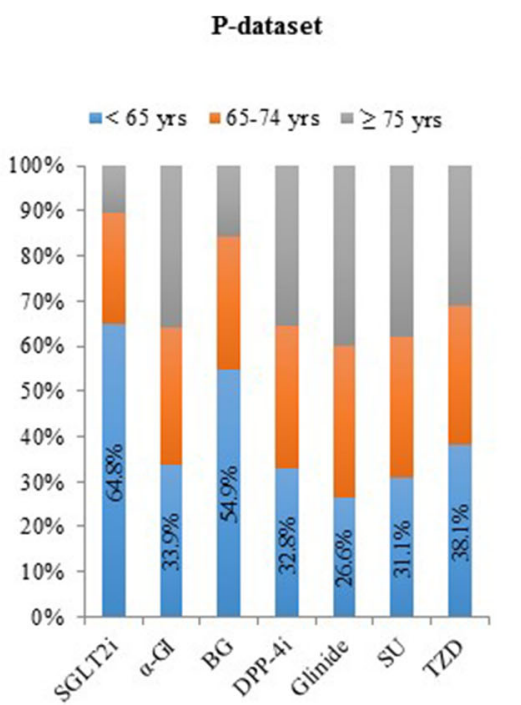

P-dataset

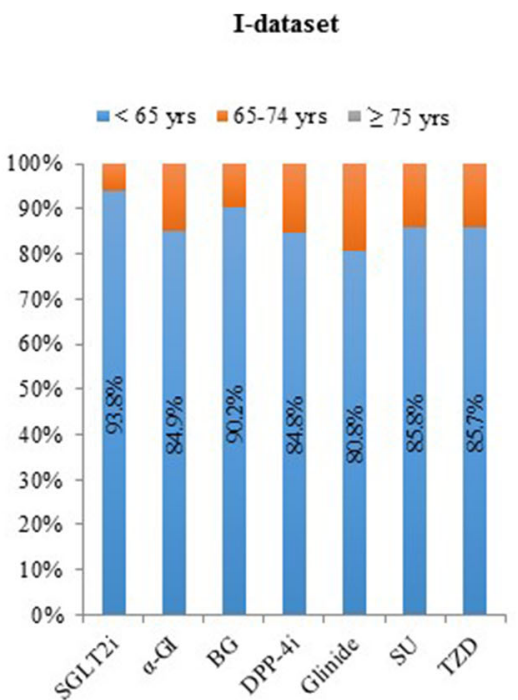

Fig. 2 Age distribution of SGLT2i users and other OAD users at index. $H$-dataset a hospital-based administrative database constructed from data for inpatients and outpatients from 287 diagnosis procedure combination (DPC) hospitals. $P$-dataset a pharmacy claims database using data from over 800 pharmacies nation-wide which provided a coverage of approximately $2 \%$ of all outpatient

the other OAD cohorts. A window period of - 30 days was allowed for the collection of baseline clinical values [body mass index (BMI), glycated hemoglobin (HbA1c), estimated glomerular filtration rate (eGFR)]. If there were multiple values within this period, the closest one to the index date was chosen. Comorbidities were coded according to the Elixhauser Comorbidity Index (ECI) [23, 24] and scored as previously reported [25]. The prevalence of hypertension and hypercholesterolemia were assessed based on the ICD-10 diagnosis code (I10.x for hypertension and E78.x for hypercholesterolemia) and prescriptions for these conditions during the preindex period (YJ codes starting with 214 for hypertension and 218 for hypercholesterolemia). Diabetes-related complications were evaluated using the Diabetes Complication Severity Index (DCSI) [26], which identified seven complications: CVD, nephropathy,

\section{OAD cohorts}

prescriptions. I-dataset an insurance claims database containing medical and prescription claims of 3.8 million employees and their dependents that were mostly aged $\leq 65$ years. $\alpha-G I$ alpha-glucosidase inhibitors, $B G$ biguanides, $D P P-4 i$ dipeptidyl peptidase- 4 inhibitors, $O A D$ oral antidiabetic drug, $S G L T 2 i$ sodium glucose co-transporter-2 inhibitors, $S U$ sulfonylureas, $T Z D$ thiazolidinediones

retinopathy, cerebrovascular disease, neuropathy, peripheral vascular disease and metabolic disease (ketoacidosis). The DCSI was originally developed using the ICD ninth revision (ICD-9) codes and laboratory test results, but ICD-10 codes [27, 28] with adaptations for Japan-specific standardized diagnosis codes and serum creatinine results (where available) were used in this study (see ESM Table 2 for a list of diagnosis codes).

\section{Statistical Analysis}

We analyzed the data in each dataset separately. Continuous data were presented as the mean and standard deviation (SD) or as the median and inter-quartile range (IQR), as appropriate, and categorical data were presented as proportion. Prescription site was summarized according to the number of beds ( $\geq 20$ beds as hospital and $<20$ beds as clinic). 
Changes over time in prescription site, prescribing specialty and age at index were aggregated quarterly by index date. All analyses were conducted for each dataset separately using SAS $^{\circledR}$ Studio Release 3.5 (SAS Institute Inc., Cary, NC, USA).

\section{RESULTS}

\section{Prescription Site, Prescribing Specialty and Patient Demographics}

In total, 176,355 patients in the H-dataset, 98,361 patients in the P-dataset, and 37,786 patients in the I-datasets were included in the analysis (Fig. 1). Table 1 shows the characteristics of SGLT2i users and of users of other OADs. Overall, $38.8 \%$ of the patients in the P-dataset and $68.7 \%$ of those in the I-dataset received their index prescriptions from clinics. Age/gender distribution were similar between patients in the H-dataset (only included hospital patients) and those in the P-dataset (included both clinic and hospital patients). The H-dataset contained more prescriptions initiated by diabetologists than did the P-dataset. Compared with users of other OADs, SGLT2i users and TZD users tended to have more prescriptions initiated from the clinics. Over the study period, there was a decreasing trend in SGLT2i prescriptions initiated from the clinics (ESM Fig. 1). OAD prescriptions were most frequently initiated by generalists across all cohorts (Table 1 ). Notably, the number of SGLT2i prescriptions initiated in the cardiology department increased slightly over the study period (ESM Fig. 2).

There were no differences in gender distribution between SGLT2i users and users of other OADs in each dataset, but SGLT2i users were the youngest among the OAD cohorts (Table 1; Fig. 2). Over the study period, there was an increasing trend in mean age at index among SGLT2i users (Fig. 3), with an increase in the proportion of patients aged $\geq 65$ years in all datasets (ESM Fig. 3).
Fig. 3 Mean age at index during the study period in the a H-dataset, b P-dataset, and $\mathbf{c}$ I-dataset. H-dataset a hospitalbased administrative database constructed from data for inpatients and outpatients from 287 diagnosis procedure combination (DPC) hospitals. $P$-dataset a pharmacy claims database using data from over 800 pharmacies nation-wide which provided a coverage of approximately $2 \%$ of all outpatient prescriptions. I-dataset an insurance claims database containing medical and prescription claims of 3.8 million employees and their dependents that were mostly aged $\leq 65$ years. $\alpha$ - $G I$ alpha-glucosidase inhibitors, $B G$ biguanides, $D P P$ $4 i$ dipeptidyl peptidase- 4 inhibitors, $S G L T 2 i$ sodium glucose co-transporter-2 inhibitors, $S U$ sulfonylureas, $\quad T Z D$ thiazolidinediones

\section{Clinical Characteristics}

The comorbidities and diabetes-related complications at index among SGLT2i users and users of other OADs in the $\mathrm{H}$ - and I-datasets are summarized in Table 2. The median ECI score was lower in both SGLT2i and BG users than in users of other OADs in the H-dataset but was comparable across all OAD users in the I-dataset. A higher prevalence of hypertension was noted in SGLT2i users in the I-dataset and a higher prevalence of hypercholesterolemia was noted in SGLT2i users in both the $\mathrm{H}$ - and I-datasets compared with users of other OADs. The median DCSI score was similar across all OAD cohorts in both the $\mathrm{H}$ - and I-datasets. There were no distinct differences in the prevalence of complications between SGLT2i users and users of other OADs in both of these datasets.

The body weight, BMI, HbA1c and eGFR levels at index in SGLT2i users and users of other OADs in the $\mathrm{H}$ - and I-datasets are shown in Table 3. SGLT2i users in the H-dataset had the highest mean body weight, BMI and HbA1c levels among the OAD cohorts (Table 3 ). The mean BMI level was also highest in SGLT2i users in the I-dataset. All OAD cohorts in both datasets had median eGFR levels of $>60 \mathrm{~mL} / \mathrm{min} /$ $1.73 \mathrm{~m}^{2}$. 

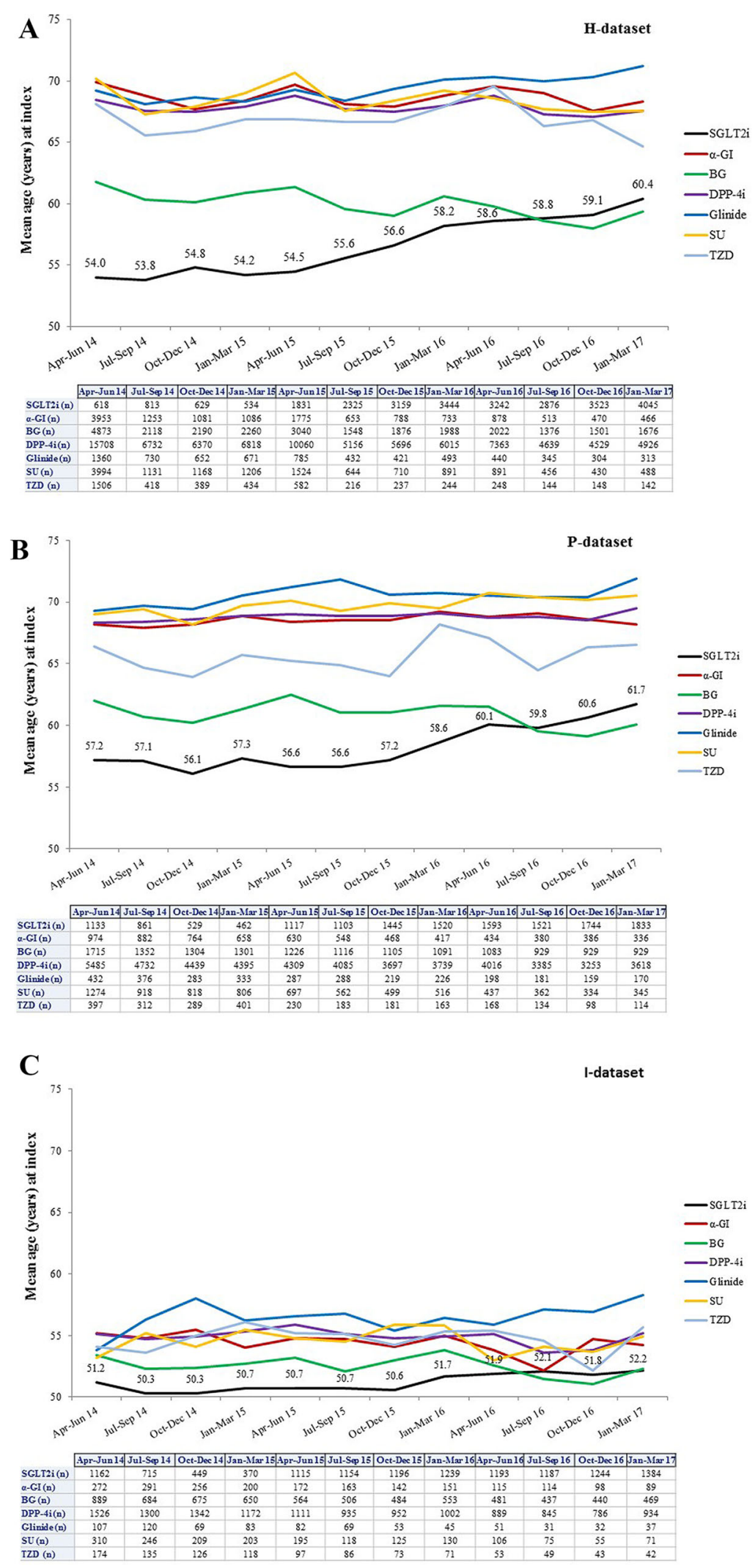


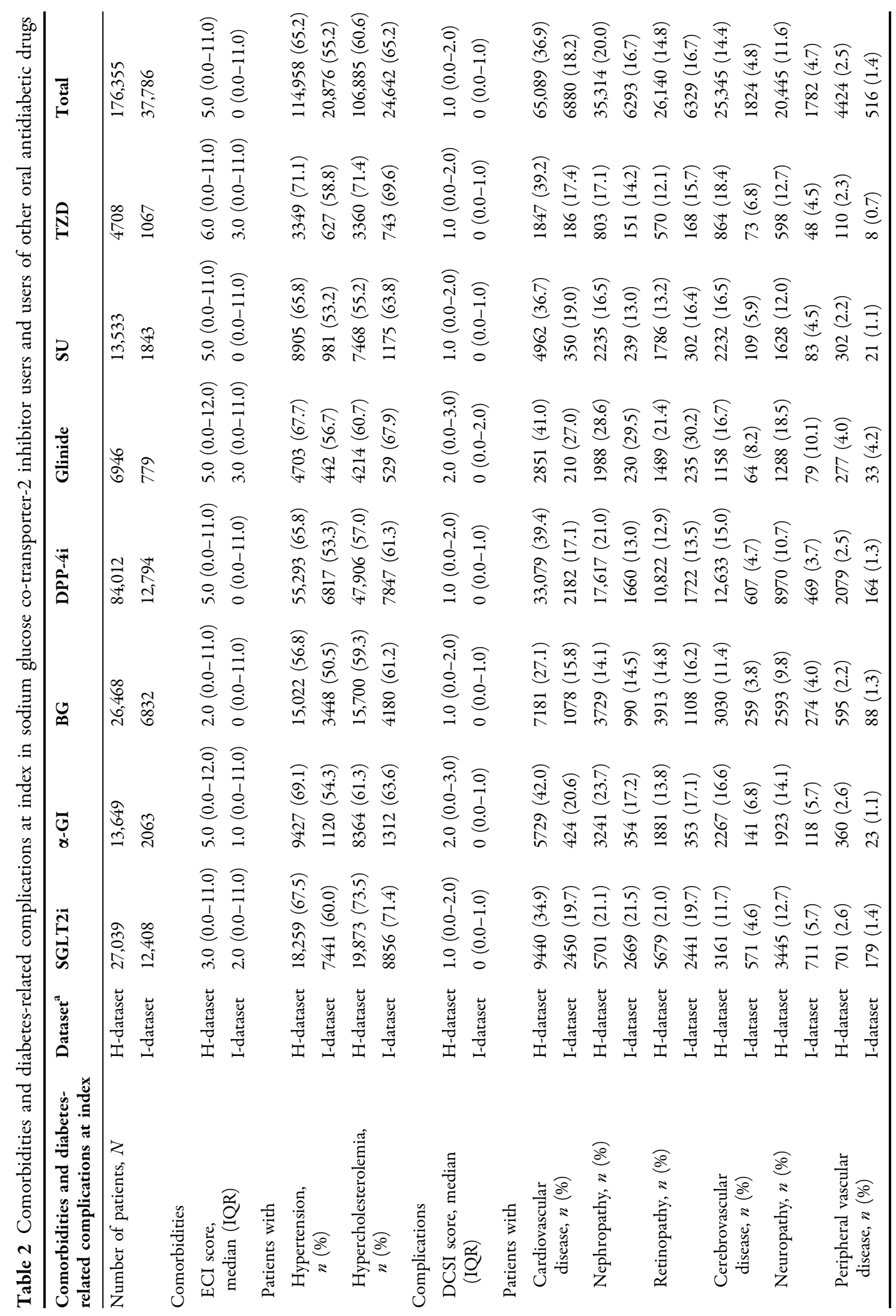




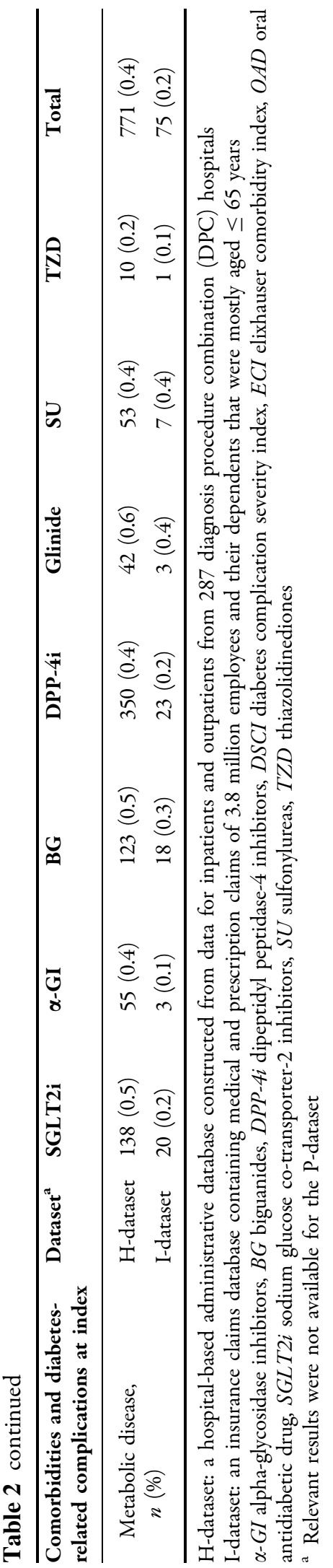

\section{DISCUSSION}

This study, to the best of our knowledge, is the largest study to characterize new users of SGLT2i using data from multiple databases in Japan. The results of this study showed that SGLT2i new users tended to be younger and to have higher BMI levels and a higher prevalence of hypertension and hypercholesterolemia at index than did other new users of OADs during the first 3 years of the launching of SGLT2i use in Japan. SGLT2i new users also tended to be heavier and to have higher HbA1c levels than users of other OADs. SGLT2i were initially prescribed more frequently to younger patients and subsequently gradually introduced to elderly patients. SGLT2i prescriptions were initiated more frequently from clinics and then progressively also from hospitals. Notably, the number of SGLT2i prescriptions initiated in the cardiology department increased slightly over time. Hankins et al. [29] conducted a similar database study between November 2012 and April 2014 to compare early users of dapagliflozin with users of other OADs in Germany. The dapagliflozin users were younger and heavier and had higher HbA1c levels than did users of other OADs [29], similar to the SGLT2i new users in the present study. However, the German study did not report any differences in the prevalence of hypertension and hypercholesterolemia between dapagliflozin users and the users of other OADs during the study period [29].

In this study, we observed that SGLT2i users tended to have a higher prevalence of hypertension and hypercholesterolemia at initiation compared with users of other OADs. In addition, a slight increase in SGLT2i prescription initiated from the cardiology department was noted over the study period. A recent metaanalysis demonstrated reductions in hospitalization for heart failure and delayed progression of renal disease in patients with T2DM treated with SGLT2i, regardless of existing atherosclerotic CVD or history of heart failure [30]. Therefore, SGLT2i may also be gradually introduced to patients without an established risk of CVD. 


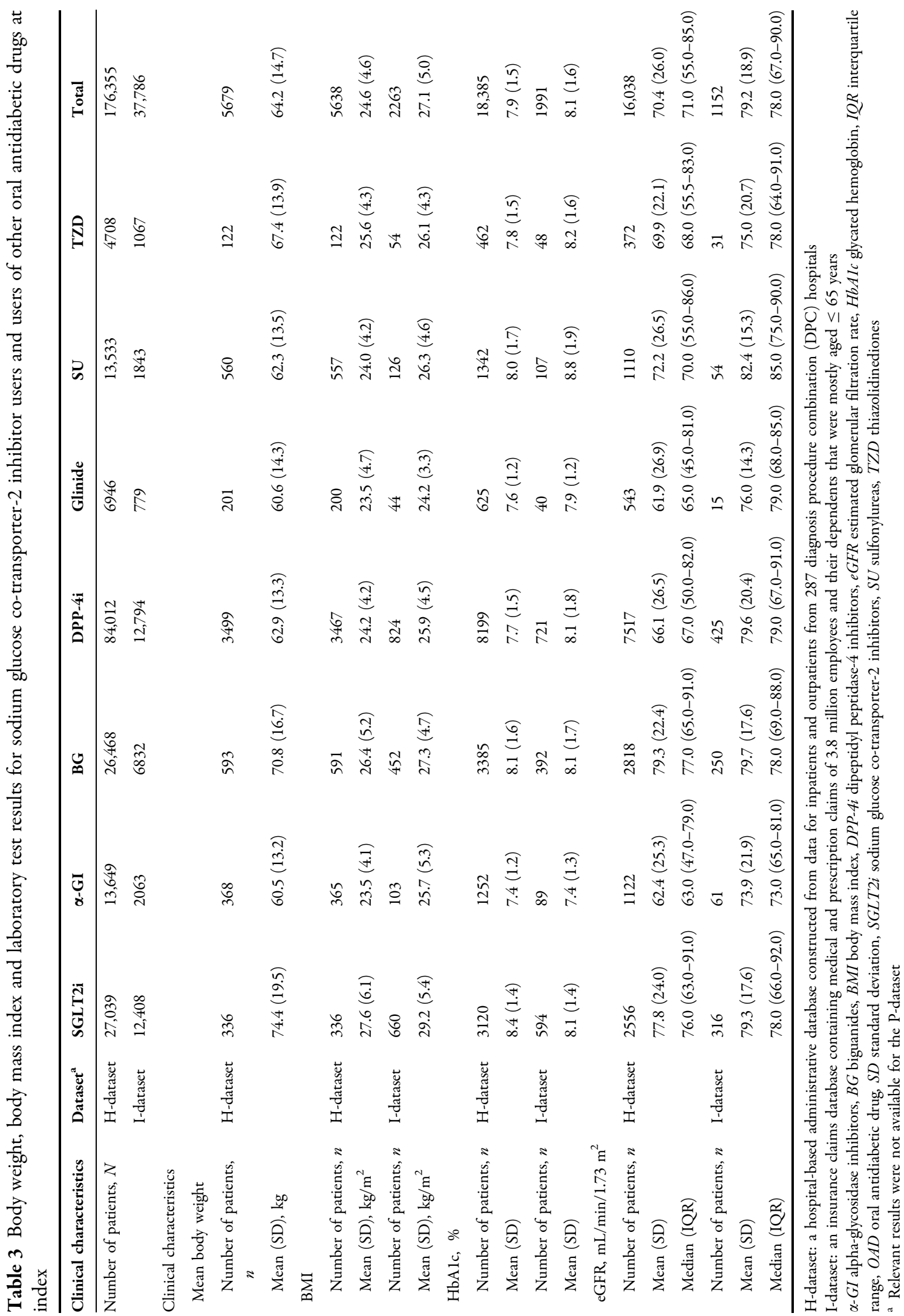


Clinical trials have demonstrated a weight reduction benefit in addition to improved glycemic control in patients with T2DM treated with SGLT2i [8-14]. In this study, we observed that SGLT2i users tended to be heavier than the users of other OADs at treatment initiation, suggesting that SGLT2i may be considered for patients who have concerns about weight gain with diabetes treatment, although this result may be due to the new users of SGLT2i being younger than the users of other OADs. SGLT2i reduce blood glucose levels via an insulin-independent mechanism and are suitable for patients with T2DM regardless of the level of beta-cell function and the degree of insulin resistance [7]. The present study showed that SGLT2i users tended to have higher HbA1c levels than other the users of OADs at treatment initiation. Considering the unique mode of action of SGLT2i, it may be that SGLT2i were considered for patients who were at later stage of the diabetes or for those whose blood sugar was inadequately controlled. Further studies on the drug utilization patterns in SGLT2i users will provide more information on physicians' prescribing behavior.

There were some limitations to this study. While we sought 'new' users of an OAD, the index OAD prescription in the $\mathrm{H}$ - and P-dataset may not be truly 'new', as patients may have prescriptions from other hospitals or pharmacies that could not be captured by the databases. Also, the I-dataset had mostly younger patients ( $\leq 65$ years) and the H-dataset included only DPC hospitals. These patients may not be representative of the general diabetes population. Nevertheless, results from the three databases were similar. By selecting patients with SGLT2i prescriptions ahead of the other OADs, the relative cohort sizes were not meaningful. In addition, the diagnoses of comorbidities and complications were not validated in these databases, and the prevalence may have been underestimated [31]. Further, laboratory data were available for only a limited number of patients.

\section{CONCLUSIONS}

The results of this study show that patients starting on SGLT2i differed in several demographic and clinical characteristics from new users of other OADs. While SGLT2i were prescribed more frequently to younger patients, over the study period they were gradually being used in elderly patients. In addition, there is a trend towards prescribing SGLT2i to patients at increased risk of CVD or those with poorer glycemic control. Further studies are needed to elucidate the drug utilization patterns in SGLT2i users.

\section{ACKNOWLEDGEMENTS}

Funding. Sponsorship for this study and article processing charges were funded by Astellas Pharma Inc., Tokyo, Japan. All authors had full access to all of the results in this study and take complete responsibility for the integrity of the data and accuracy of the data analysis.

Medical Writing and Editorial Assistance. Editorial assistance in the preparation of this article was provided by Tech Observer Asia Pacific Pte Ltd., Singapore. Support for this assistance was funded by Astellas Pharma Inc., Tokyo, Japan.

Authorship. All named authors meet the International Committee of Medical Journal Editors (ICMJE) criteria for authorship for this article, take responsibility for the integrity of the work as a whole, and have given their approval for this version to be published.

Disclosures. Yuichiro Ito is an employee of Astellas Pharma Inc., Tokyo, Japan. Takuya Nishimura is an employee of Astellas Pharma Inc., Tokyo, Japan. Toshifumi Sugitani is an employee of Astellas Pharma Inc., Tokyo, Japan. Tomomi Kimura is an employee of Astellas Pharma Inc., Tokyo, Japan. James Van Schyndle is an employee of Astellas US, LLC., Northbrook, USA.

Compliance with Ethics Guidelines. The Medical Affairs Japan Protocol Review Committee reviewed and approved the study 
protocol prior to study initiation. As the study only involved analysis of pre-existing data in the databases, written informed consent from the study participants was not required. This study was conducted in accordance with the guidelines for Good Pharmacoepidemiology Practice [19].

Data Availability. Access to anonymized individual participant level data will not be provided for this study as it meets one or more of the exceptions described on www. clinicalstudydatarequest.com under "Sponsor Specific Details for Astellas."

Open Access. This article is distributed under the terms of the Creative Commons Attribution-NonCommercial 4.0 International License (http://creativecommons.org/licenses/ by-nc/4.0/), which permits any noncommercial use, distribution, and reproduction in any medium, provided you give appropriate credit to the original author(s) and the source, provide a link to the Creative Commons license, and indicate if changes were made.

\section{REFERENCES}

1. Charvat H, Goto A, Goto M, et al. Impact of population aging on trends in diabetes prevalence: a meta-regression analysis of 160,000 Japanese adults. J Diabetes Investig. 2015;6(5):533-42.

2. International Diabetes Federation (IDF) Western Pacific members. Japan. 2018. https://www.idf.org/ our-network/regions-members/western-pacific/ members/105-japan.html. Accessed 5 May 2018.

3. Laakso M, Kuusisto J. Insulin resistance and hyperglycaemia in cardiovascular disease development. Nat Rev Endocrinol. 2014;10(5):293-302.

4. Zheng Y, Ley SH, Hu FB. Global aetiology and epidemiology of type 2 diabetes mellitus and its complications. Nat Rev Endocrinol. 2018;14(2):88-98.

5. Guthrie RM. Evolving therapeutic options for type 2 diabetes mellitus: an overview. Postgrad Med. 2012;124(6):82-9.

6. Marin-Penalver JJ, Martin-Timon I, Sevillano-Collantes C, Del Canizo-Gomez FJ. Update on the treatment of type 2 diabetes mellitus. World J Diabetes. 2016;7(17):354-95.

7. Chao EC, Henry RR. SGLT2 inhibition-a novel strategy for diabetes treatment. Nat Rev Drug Discov. 2010;9(7):551-9.

8. Ikeda S, Takano Y, Cynshi O, et al. A novel and selective sodium-glucose cotransporter-2 inhibitor, tofogliflozin, improves glycaemic control and lowers body weight in patients with type 2 diabetes mellitus. Diabetes Obes Metab. 2015;17(10):984-93.

9. Inagaki N, Kondo K, Yoshinari T, et al. Efficacy and safety of canagliflozin monotherapy in Japanese patients with type 2 diabetes inadequately controlled with diet and exercise: a 24-week, randomized, double-blind, placebo-controlled, Phase III study. Expert Opin Pharmacother. 2014;15(11):1501-15.

10. Kadowaki T, Haneda M, Inagaki N, et al. Efficacy and safety of empagliflozin monotherapy for 52 weeks in Japanese patients with type 2 diabetes: a randomized, double-blind, parallel-group study. Adv Ther. 2015;32(4):306-18.

11. Kaku K, Maegawa H, Tanizawa Y, et al. Dapagliflozin as monotherapy or combination therapy in Japanese patients with type 2 diabetes: an open-label study. Diabetes Ther. 2014;5(2):415-33.

12. Kashiwagi A, Kazuta K, Yoshida S, Nagase I. Randomized, placebo-controlled, double-blind glycemic control trial of novel sodium-dependent glucose cotransporter 2 inhibitor ipragliflozin in Japanese patients with type 2 diabetes mellitus. J Diabetes Investig. 2014;5(4):382-91.

13. Seino $\mathrm{Y}$, Inagaki $\mathrm{N}$, Haneda $\mathrm{M}$, et al. Efficacy and safety of luseogliflozin added to various oral antidiabetic drugs in Japanese patients with type 2 diabetes mellitus. J Diabetes Investig. 2015;6(4):443-53.

14. Seino Y, Sasaki T, Fukatsu A, et al. Efficacy and safety of luseogliflozin as monotherapy in Japanese patients with type 2 diabetes mellitus: a randomized, double-blind, placebo-controlled, phase 3 study. Curr Med Res Opin. 2014;30(7):1245-55.

15. Goda M, Yamakura T, Sasaki K, Tajima T, Ueno M. Safety and efficacy of canagliflozin in elderly patients with type 2 diabetes mellitus: a 1-year postmarketing surveillance in Japan. Curr Med Res Opin. 2018;34(2):319-27.

16. Nakamura I, Maegawa H, Tobe K, Tabuchi H, Uno S. Safety and efficacy of ipragliflozin in Japanese patients with type 2 diabetes in real-world clinical practice: interim results of the STELLA-LONG TERM 
post-marketing surveillance study. Expert Opin Pharmacother. 2018;19(3):189-201.

17. Utsunomiya K, Shimmoto N, Senda M, et al. Safety and effectiveness of tofogliflozin in elderly Japanese patients with type 2 diabetes mellitus: a post-marketing study (J-STEP/EL Study). J Diabetes Investig. 2017;8(6):766-75.

18. Yokote K, Terauchi Y, Nakamura I, Sugamori H. Real-world evidence for the safety of ipragliflozin in elderly Japanese patients with type 2 diabetes mellitus (STELLA-ELDER): final results of a post-marketing surveillance study. Expert Opin Pharmacother. 2016;17(15):1995-2003.

19. Public Policy Committee, International Society of Pharmacoepidemiology. Guidelines for good pharmacoepidemiology practice (GPP). Pharmacoepidemiol Drug Saf. 2016;25(1):2-10.

20. Medical Data Vision Co. Ltd (MDV). Introducing MDV database. https://www.mdv.co.jp/solution/ pharmaceutical/english/. Accessed 27 Apr 2018.

21. Kyowa Kikaku Ltd. Medi-Trend (out-of-hospital prescription database system). Accessed at Kyowa Kikaku Ltd. at https://www.kk-kyowa.co.jp/ medical_prescription.html. Accessed 29 Aug 2018.

22. Kimura S, Sato T, Ikeda S, Noda M, Nakayama T. Development of a database of health insurance claims: standardization of disease classifications and anonymous record linkage. J Epidemiol. 2010;20(5):413-9.

23. Elixhauser A, Steiner C, Harris DR, Coffey RM. Comorbidity measures for use with administrative data. Med Care. 1998;36(1):8-27.

24. Quan H, Sundararajan V, Halfon P, et al. Coding algorithms for defining comorbidities in ICD-9-CM and ICD-10 administrative data. Med Care. 2005;43(11):1130-9.

25. van Walraven C, Austin PC, Jennings A, Quan H, Forster AJ. A modification of the Elixhauser comorbidity measures into a point system for hospital death using administrative data. Med Care. 2009;47(6):626-33.

26. Young BA, Lin E, Von Korff $M$, et al. Diabetes complications severity index and risk of mortality, hospitalization, and healthcare utilization. Am J Manag Care. 2008;14(1):15-23.

27. Glasheen WP, Renda A, Dong Y. Diabetes Complications Severity Index (DCSI) - update and ICD-10 translation. J Diabetes Complicat. 2017;31(6):1007-13.

28. Wilke T, Mueller S, Groth A, et al. Treatment-dependent and treatment-independent risk factors associated with the risk of diabetes-related events: a retrospective analysis based on 229,042 patients with type 2 diabetes mellitus. Cardiovasc Diabetol. 2015;14:14.

29. Hankins M, Tsai K, Kim J, Hammar N. Early drug use of dapagliflozin prescribed by general practitioners and diabetologists in Germany. Diabetes Res Clin Pract. 2017;125:29-38.

30. Zelniker TA, Wiviott SD, Raz I, et al. SGLT2 inhibitors for primary and secondary prevention of cardiovascular and renal outcomes in type 2 diabetes: a systematic review and meta-analysis of cardiovascular outcome trials. Lancet. 2018;393:31.

31. Yamana $H$, Moriwaki M, Horiguchi $H$, et al. Validity of diagnoses, procedures, and laboratory data in Japanese administrative data. J Epidemiol. 2017;27(10):476-82. 